\title{
Human Temporomandibular Joint Motion: A Synthesis Approach for Designing a Six-Bar Kinematic Simulator
}

\author{
Michel Demuynck \\ Aidin Delnavaz \\ Department of Mechanical Engineering Department of Mechanical Engineering \\ École de technologie supérieure \\ Université du Québec \\ École de technologie supérieure \\ Université du Québec \\ QC H3C 1K3, Montréal, QC, Canada QC H3C 1K3, Montréal, QC, Canada \\ Email: michel.demuynck.1@ens.etsmtl.caEmail: aidin.delnavaz@ens.etsmtl.ca
}

\author{
Jérémie Voix \\ Department of Mechanical Engineering \\ École de technologie supérieure \\ Université du Québec \\ QC H3C 1K3, Montréal, QC, Canada \\ Email: jeremie.voix@etsmtl.ca
}

The human earcanal is a home of many in-ear devices including hearing aids, earphones, hearing protections and earplugs. This canal-type home has a moving neighbor called Temporomandibular Joint (TMJ) that slightly deforms its shape due to the jaw movements. These cyclic deformations of earcanal have a serious impact on the positioning, comfort and functioning of its fitted devices and contains significant amount of energy to harvest. Although the TMJ movements and the earcanal deformations have been the subject of many researches, their mutual actions are not still fully understood. This paper presents the development of a six-bar kinematic TMJ simulator capable of replicating the complicated motion of the jaw. Indeed, the underlying development relies on a two-phase mechanism design algorithm to numerically optimize and analytically synthesize linkage mechanisms for which the classical optimization approaches cannot return a converged solution. The proposed algorithm enables the design of a kinematic simulator to generate the TMJ path with an average error as low as $1.65 \%$ while respecting all the hinge axis parameters of the jaw. This algorithm can be subsequently used to solve nonlinear complex linkage synthesis problems. Finally, the developed kinematic simulator can be ultimately used to further investigate the TMJ-earcanal interactions.

\section{Introduction}

The human body can perform many movements using its articulations that connect to the bones. One of the most interesting articulation located in the area of the head is the temporomandibular joint (TMJ) which is involved in many jaw activities including eating, speaking, chewing, laughing, etc. TMJ is the bony protuberance of the jaw called mandibular condyle which is hosted within the fossa of the temporal bone of the skull. Many studies showed the mechanical influence of TMJ onto its surrounding soft tissues during opening and closing the jaw which is resulting in geometrical deformations of the earcanal, also called earcanal dynamic movement [1]. The earcanal movements caused by TMJ activity possess some kinetic energy which can be harvested to power electronic circuit of in-ear devices. Energy harvesting from inside the earcanal has been studied in recent years and a few energy scavenger prototypes have been developed so far. However, the exact earcanal deformation magnitude and the amount of energy available from TMJ activity is yet to be investigated. Given the small size and the curvy shape of the earcanal, the TMJ location is hard to access for in vivo measurements and the biomechanical interactions between the TMJ and the earcanal has not yet been fully known. So far, two methods have been proposed to quantify the earcanal dynamic movement: 1) an experimental method consisting of a hydraulic system which transform the earcanal deformation into a measurable pressure variation in a water column $[1,2]$ and 2) an analytical method based on the bending beam theory to model the earcanal wall deformation [3]. Although the obtained results are encouraging, the extensive level of simplification used in prior modelings questions the veracity of the results and necessitate the precise modeling of the TMJ-earcanal interactivity and interactions. 
The TMJ activity and the earcanal shapes and deformations have been separately investigated by many groups of researchers to date. The complex kinematic of the TMJ has been studied either in 2D or 3D space, by focusing on the incisor path [4-7], the mandibular condyle path [8-11] or the mandibular helical axis path $[12,13]$. These studies provide more insight to the mechanical behavior of the TMJ in different ranges of its motion including elevation/dropping, lateral deviation and protraction/retraction of the jaw. The forces applied by the TMJ during the mastication has also been the topic of many experimental researches. Some types of jaw motion simulators, replicators or robots have been developed to measure the TMJ loads during the mastication. All these robotic platforms mimic both kinematics and dynamics of the jaw movement either in a simple 1 degree of freedom (1-DOF) model [14], or a 3-DOF model [15-17] or more recently in a complicated 6-DOF model $[18,19]$. In addition, the dental articulators which are currently in use by the dentists and the orthodontists enable the reproduction of some or all the movements of the mandible (lower jaw) in relation to the maxilla (upper jaw) to assist in the accurate fabrication of prosthodontic or orthodontic appliances.

On the other side, the earcanal geometry and its associated dynamic movements have been the subject of many researches by audiologists and hearing conservationists to improve the process of ear impression required to make customized hearing aids and custom-fitted earplugs [20-22]. To the best of the authors' knowledge, a comprehensive study involving both TMJ and earcanal as well as their mutual interactions is still missing in the literature. To this end, a TMJearcanal simulator based on a planar linkage mechanism is required to investigate how $\mathrm{TMJ}$ deforms the earcanal and how the earcanals reacts to the TMJ forces during jaw joint activity.

One of the best candidates to realize the TMJ-earcanal simulator is a planar linkage mechanism which are widely used to design anatomic prostheses, humanoid robots or exoskeletons capable of mimicking the human body joints motion. As compared with servo-controlled devices such as machine tool arms, the proposed linkage mechanism control design has the clear advantage of being much less expansive and much simpler to duplicate as a research tool: the integration of the proposal mechanics with an electrical motor could reproduce the TMJ anatomical behavior with a rotational input movement. As compared with other motiontransforming mechanisms such as cam-and-roller or rackand-pinion ones, linkage mechanisms are capable of offering more complex types of motion while requiring a simpler architecture and fewer articulations. Also, the theory of such mechanisms is well known which makes their synthesis, design and analysis much easier to implement using inverse dynamics solution. Path generation and motion generation are two important types of problem in the mechanism synthesis. Path generation is the control of the mechanism coupler point to pass through a desired set of discrete points while the motion generation is the control of a coupler as a solid body in both position and orientation to move through some prescribed positions. Given a very limited number of design parameters for the linkage mechanisms, the optimization is normally required for the mechanism synthesis.

Optimal synthesis of mechanisms can be found in many biomedical prosthesis design. For example, a cross four-bar linkage mechanism to reproduce the movement of the middle finger of the human hand has been presented in [23]. Such mechanism is optimized according to the bending angle of the second phalanx by minimizing the mean quadratic error between the generated values and the desired ones. An optimized Stephenson six-bar linkage mechanism capable of reproducing the trajectory of the ankle joint according to the hip one for a human walking exoskeleton has been developed in [24]. This study proposes a 11-point path synthesis combined with an optimization process allowing the mechanism to pass through 60 accuracy points by minimizing the difference between the coupler point and the data points collected from a motion capture sensor. Another kind of six-bar linkage mechanism design has been proposed for a three-jointed finger mechanism [25]. The position of the coupler and its orientation compared with the data of the distal segment of the human finger are minimized with the least squares criteria.

The purpose of this study is to propose a biologicallyinspired two-phase synthesis approach to design a six-bar linkage mechanism (SBLM) reproducing the TMJ movement while interacting with the earcanal. The feasibility to design a simplified-architecture mechanism that is only driven by the hinge axis joints is evaluated in this paper. The results of the paper can be adapted for the use in several fields including but not limited to the design of dental articulators, surgical mandibular implants or devices used to cure TMJ disorders. Also, other studies on the TMJ-earcanal interaction such as those focused on the earplug comfort [26], hearing aid retention problem $[27,28]$ or energy harvesting from earcanal dynamic movement [29-31] can substantially benefit from the result of this study.

\section{Biomechanics of the TMJ}

Studying the jaw joint movements and its influence on the earcanal deformations requires some definitions to describe the TMJ movements and constraints. The hinge axis is defined as an imaginary line which is perpendicular to the mid-sagittal plane of the head, passing through the centers of both mandibular condyles as shown by a blue point in Fig. 1a. In most studies, the hinge axis is a reference to measure the kinematic parameters of the jaw movements like the mandibular condyle path (yellow dashed line), the incisor path $s_{\text {incisor }}\left(\right.$ red line) and the sagittal rotation $\Phi_{\text {hinge }}$ (red arc) as illustrated in Fig. 1a.

The geometrical path of the hinge axis represents the curvilinear translation of the mandibular condyle. The following mathematical function has been proposed by Peck as a boundary condition for the mandibular condyle path during the jaw opening [32] 


$$
y(x)=5 \cos \left(\frac{\pi}{13} x\right)-5
$$

where $x$ denotes the coordinate along the anteroposterior (A-P) axis and $y$ denotes the coordinate along the supero-inferior (S-I) axis. In fact, this formula represents the shape of the temporal fossa of the skull bone, which is the superior anatomical bound of the mandibular condyle movement. As shown in Fig. 1a, the proposed profile is oriented with respect to the anatomical planes attached to the human head by a $40^{\circ}$ rotation with respect to the occlusal plane and a $20^{\circ}$ rotation with respect to the mid-sagittal plane. Moreover, the profile length corresponds to a 16-millimeters horizontal translation of the mandibular condyle along the A-P axis.

The hinge axis is also the axis around which the jaw performs its rotation during mouth opening. This rotation induces a curvilinear translation of the incisor. Several studies has been focused on the relations between the incisor and the condylar paths. For example, a $32.05 \pm 0.90^{\circ}$ sagittal rotation of the jaw can be associated to a $48.60 \pm 1.03 \mathrm{~mm}$ curvilinear translation of the incisor by performing an optoelectric tracking of the jaw [4].

For this preliminary study, it is assumed that the occlusal plane is horizontal, the hinge axis opening rotation of the jaw $\Phi_{\text {hinge }}$ is fixed at $32^{\circ}$ in clockwise rotation and the mouth opening movement is symmetric between the left and right TMJ. The target path is defined as a set of discrete positions resulting from the function presented in Eqn. (1) after performing the anatomical transformations $\left(40^{\circ}\right.$ orientation according to the occlusal plane and $16 \mathrm{~mm}$ truncation of the translation path). Since the study frame is based on the parasagittal plane passing through the mandibular condyle center, the angular orientation of the temporal fossa profile according to the mid-sagittal plane is not considered. The mandibular condyle is assumed going back-and-forth along the profile described by the anatomical-adjusted function of Peck. The curvilinear length of the incisor path $s_{\text {incisor }}$ is supposed to be $50 \mathrm{~mm}$.

\section{One-phase optimization approach: Motion genera- tion synthesis}

Several mechanisms can be used for continuous path generation passing through a set of specified discrete positions. The mechanism should be able to: (1) perform a planar (2D) motion as the target path is a $2 \mathrm{D}$ curve, (2) to be driven by one input signal to have the simplest possible control, and (3) favor pivoted links over slider links as the design and implementation of pivoted links are technically much simpler than those of slider links. Therefore, the four bar linkage mechanism (FBLM-1), composed only of pivoted or rotating articulations is best candidate for this study. Figure 2 schematically shows the FBLM-1 model in its initial (solid line) and intermediate (dashed line) positions after an arbitrary rotation of $\Delta \theta_{\mathrm{A}}$ for the input link.
As illustrated in Fig. 2, 11 design variables can be identified for the FBLM-1: the lengths of all the links $l_{1}, l_{2}, l_{3}$, $l_{4}$ and $l_{\mathrm{C}}$, the global orientation of the mechanism by angle $\alpha$ with respect to the reference A-P axis, the coupler angle $\gamma$, the initial angular position of the input link $\theta_{\mathrm{A}, 0}$, the angular variation of the input angle $\Delta \theta_{\mathrm{A}}$, and the coordinates $\left[x_{\mathrm{A}}, y_{\mathrm{A}}\right]$ of the point $A$. The global $(x, y)$ and local $(u, v)$ frames are orthogonal coordinate systems fixed in point $A$ whose first axes are aligned with the reference A-P axis and link $l_{1}$ respectively as shown in Fig. 2.

The optimized configuration of the FBLM-1 model should generate a path at point $C$ that fits to the target path as close as possible. To this end, the coordinates of the coupler point $C$ should be expressed as a function of the design variables in the global coordinate system by:

$$
\left\{\begin{array}{l}
x_{C}=x_{A}+l_{2} \cos \left(\theta_{A}+\alpha\right)+l_{C} \cos \left(\gamma+\theta_{P}+\alpha\right) \\
y_{C}=y_{A}+l_{2} \sin \left(\theta_{A}+\alpha\right)+l_{C} \cos \left(\gamma+\theta_{P}+\alpha\right)
\end{array}\right.
$$

It is therefore necessary to express the design parameter $\theta_{\mathrm{P}}$ as a function of the design variables by using the Freudenstein's method [33]. Projecting the geometric loop in Fig. 2 onto the directions $\vec{u}$ and $\vec{v}$ yields:

$$
\left\{\begin{array}{l}
l_{2} \cos \left(\theta_{A}\right)+l_{3} \cos \left(\theta_{P}\right)-l_{4} \cos \left(\theta_{B}\right)-l_{1}=0 \\
l_{2} \sin \left(\theta_{A}\right)+l_{3} \sin \left(\theta_{P}\right)-l_{4} \sin \left(\theta_{B}\right)=0
\end{array}\right.
$$

By eliminating $\theta_{B}$ in Eqns. (3) and using $K_{1}=\frac{l_{1}}{l_{2}}, K_{2}=$ $\frac{l_{1}}{l_{4}}, K_{3}=\frac{l_{4}^{2}-l_{1}^{2}-l_{2}^{2}-l_{3}^{2}}{2 l_{2} l_{3}}$, one can obtain:

$$
\begin{aligned}
& K_{1} \cos \left(\theta_{P}\right)+K_{2} \cos \left(\theta_{A}\right)+K_{3} \\
& \quad=\cos \left(\theta_{P}\right) \cos \left(\theta_{A}\right)+\cos \left(\theta_{P}\right) \cos \left(\theta_{A}\right)
\end{aligned}
$$

Then, by using the half-angle entities in Eqn. (4), a quadratic polynomial of unknown $\tan \left(\frac{\theta_{\mathrm{P}}}{2}\right)$ can be expressed as:

$$
A \tan ^{2}\left(\frac{\theta_{P}}{2}\right)+B \tan \left(\frac{\theta_{P}}{2}\right)+C=0
$$

in which:

$$
\begin{aligned}
& A=\left(1+K_{2}\right) \cos \left(\theta_{A}\right)-K_{1}+K_{3}, \\
& B=-2 \sin \left(\theta_{A}\right), \\
& C=K_{1}+\left(K_{2}-1\right) \cos \left(\theta_{A}\right)+K_{3}
\end{aligned}
$$




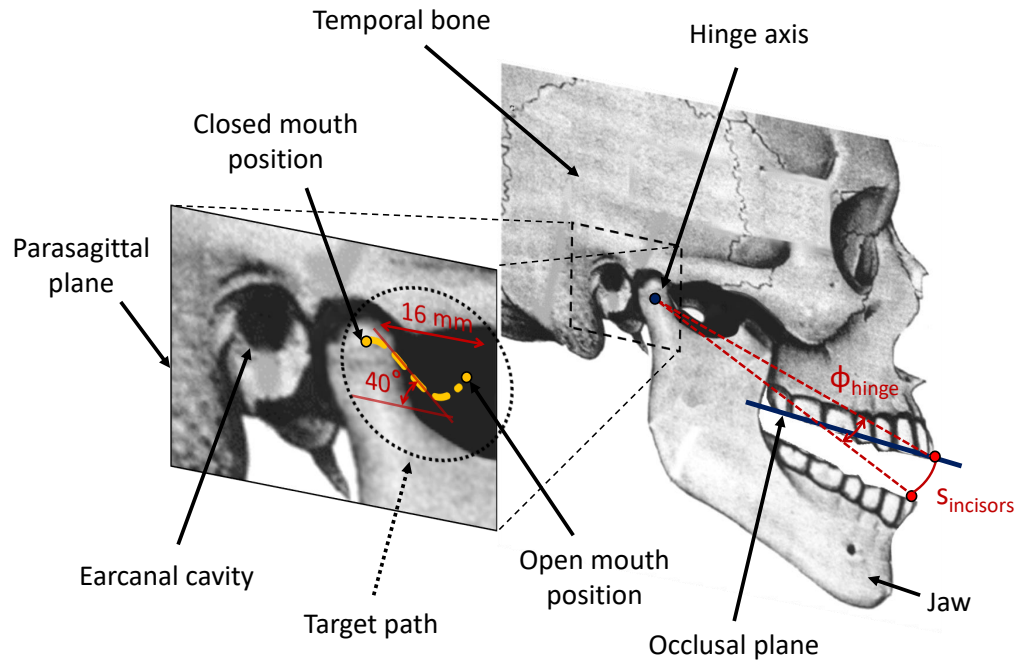

(a)

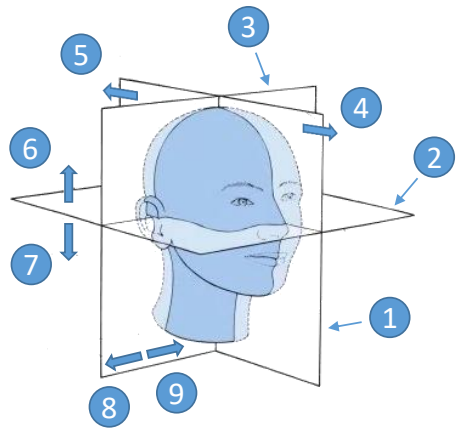

(b)

Fig. 1: (a) Anatomical setting of the TMJ model for the right side; and (b) Anatomical planes and axis associated with the head: (1) median or mid-sagittal plane, (2) horizontal or transverse plane, (3) frontal or coronal plane, (4) anterior axis, (5) posterior axis, (6) superior axis, (7) inferior axis, (8) lateral axis, and (9) medial axis.

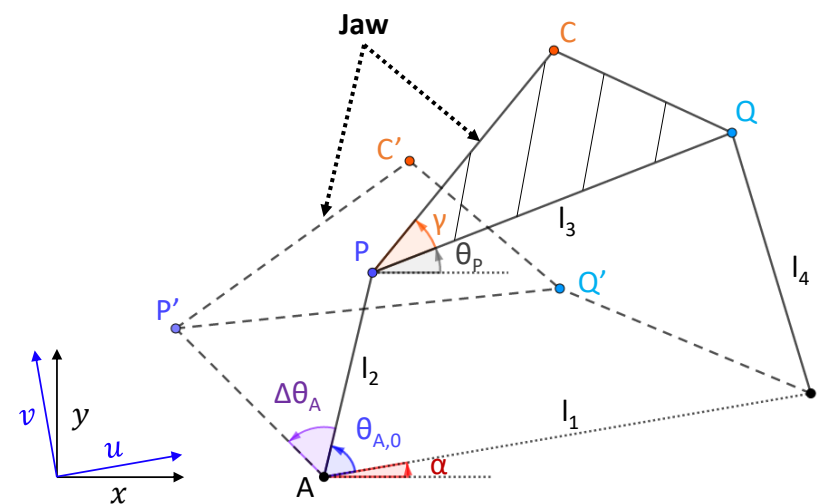

Fig. 2: Schematic representation of the FBLM-1 in its initial (solid line) and intermediate (dashed lines) positions

Finally, $\theta_{\mathrm{P}}$ is deduced from the solution of Eqn. (5):

$$
\theta_{P_{1,2}}=2 \arctan \left(\frac{-B \pm \sqrt{B^{2}-4 A C}}{2 A}\right)
$$

Equation (6) potentially provides 2 solutions, each corresponds to a specific configuration of the mechanism: ' + ' represents the solution of the open configuration (none of the links are crossed), whereas '-' corresponds to the crossed configuration (two links cross over each other). The optimization process takes into account both configurations. By replacing Eqn. (6) into Eqns. (2), the coordinates of the point $C$ are expressed as a function of the design variables.

For the optimization process, the target path is modeled as an interpolation of $N=50$ discrete points. Each of these points is compared to its associated generated point $C_{i}$ which corresponds to the angle value of $\theta_{\mathrm{A}, \mathrm{i}}$. The performance of the mechanism synthesis is evaluated based on the root-mean-square error (RMSE) between the target and the generated path normalized to the length of the displacement in the target zone $s_{\text {target }}$. Therefore, the optimum design is obtained by minimizing the following objective function:

$$
\varepsilon=\frac{\sqrt{\frac{1}{N} \sum_{i=1}^{N}\left[\left(x_{\text {target }, \mathrm{i}}-x_{C, i}\right)^{2}+\left(y_{\text {target }, \mathrm{i}}-y_{C, i}\right)^{2}\right]}}{s_{\text {target }}}
$$

The optimized FBLM-1 model would be physically realizable and biomechanically capable of reproducing the movement of the jaw if the following constraints are satisfied:

Constraint \#1: The size of the links should remain in an acceptable range in order to minimize the space requirement;

$$
15 \mathrm{~mm} \leq l_{i} \leq 150 \mathrm{~mm} \quad \forall i \in\{1,2,3,4\}
$$

Constraint \#2: Grashof condition should be imposed to ensure the smooth movement of the mechanism in its operating range including the entire target curve;

$$
\begin{aligned}
& 2 \min \left(\left\{l_{1}, l_{2}, l_{3}, l_{4}\right\}\right)+2 \max \left(\left\{l_{1}, l_{2}, l_{3}, l_{4}\right\}\right) \\
&-\left(l_{1}+l_{2}+l_{3}+l_{4}\right) \leq 0
\end{aligned}
$$

Constraint \#3: Link 2 is the input crank and should be capable of rotating a full $360^{\circ}$ without any restriction or 


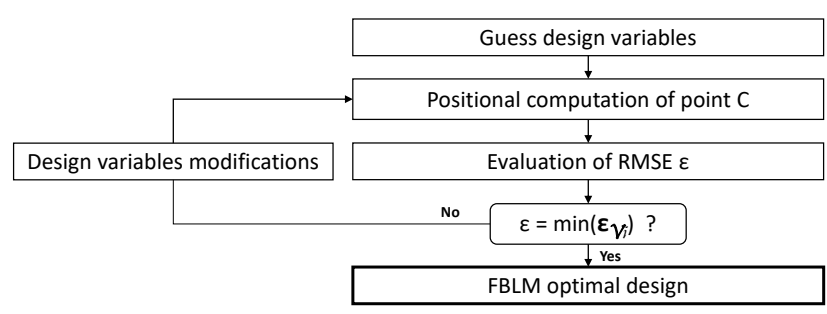

Fig. 3: Flowchart of the iterative process for solving the onephase optimization problem of FBLM-1 model. $\varepsilon_{V_{i}}$ : set of RMSE for the neighborhood of evaluation points at iteration ' $i$ '.

toggle position. Therefore, link 2 should be the shortest link in the mechanism and its length $l_{2}$ should be the smallest among links;

$$
l_{2} \leq l_{i} \quad \forall i \in\{1,3,4\}
$$

Constraint \#4: The incisor movement is modeled by the point $P$ in Fig. (2), so the curvilinear path of the point $\mathrm{P}$ should be equal to $s_{\text {incisor }}$;

$$
l_{2} \Delta \theta_{A}=s_{\text {incisor }}=50 \mathrm{~mm}
$$

Constraint \#5: The coupler should perform the biomechanical rotation $\Phi_{\text {hinge }}$ of the jaw;

$$
\Delta \theta_{P}=\Phi_{\text {hinge }}=-32^{\circ}
$$

The fmincon function with the Sequential Quadratic Programming (SQP) algorithm in the Optimization Toolbox of MATLAB (The MathWorks, Inc., Natick, MA, United States) is chosen as it is able to keep running the solving process of a nonlinear objective function even if the intermediate values are non-real. fmincon receives some input parameters including an initial set of the design variables. Therefore, it is necessary to run the optimization process by using different initial guesses to enlarge the design space. The solving process of the one-phase optimization approach is resumed in Fig. 3.

Ten iterations of the process are performed in this study for each open- and crossed-configuration of the FBLM-1 model. Finally, the optimized model is chosen based on the lowest RMSE criterion. Among the 20 results, the lowest RMSE is $\varepsilon=3.12 \%$ for the open configuration with the optimized set of design variables detailed in Table 1. Figure 4 shows the optimized generated path (dashed line) compared to the target path (solid line).

Although the optimized generated path can follow the target curve, the difference between two curves is quite considerable as seen in Fig. 4. The general shape of the generated path is not curvy enough and does not exhibit an inflec-
Table 1: Optimized values of the design variables for the FBLM-1 model

\begin{tabular}{c|cc}
\hline$l_{1}$ & 123.45 & $\mathrm{~mm}$ \\
\hline$l_{2}$ & 106.38 & $\mathrm{~mm}$ \\
\hline$l_{3}$ & 150 & $\mathrm{~mm}$ \\
\hline$l_{4}$ & 150 & $\mathrm{~mm}$ \\
\hline$l_{C}$ & 143.72 & $\mathrm{~mm}$ \\
\hline$\alpha$ & $79.49^{\circ}$ & \\
\hline$\gamma$ & $0^{\circ}$ & \\
\hline$\theta_{A, O}$ & $206.36^{\circ}$ & \\
\hline$\Delta \theta_{A}$ & $25.31^{\circ}$ & \\
\hline$x_{A}$ & 69.61 & $\mathrm{~mm}$ \\
\hline$y_{A}$ & -1.06 & $\mathrm{~mm}$ \\
\hline
\end{tabular}

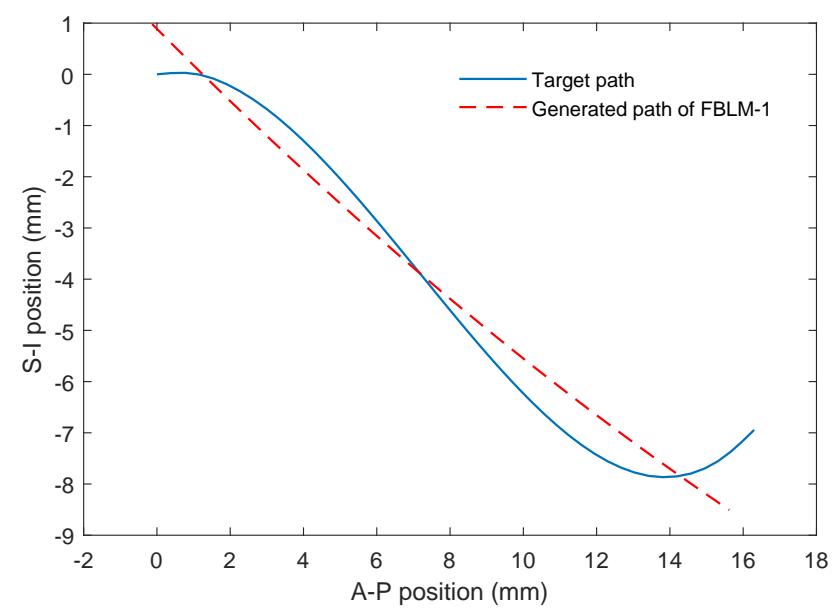

Fig. 4: Optimized generated path for the FBLM-1 model compared with the target path. Closed jaw position corresponds to the origin of the coordinate system.

tion point. Therefore, the first-order optimality value is relatively high and equals to 192.77 . Also, the maximum constraint violation value equal to 27.68 confirms that some constraints are violated in the optimization process. These parameters indicate that the FBLM-1 model is not performing well and is overconstrained. Therefore, it is necessary to relax some constraints or modify the architecture of the mechanism to split the constraints between several links. Twophase hybrid optimization approach presented in the following section aims at improving the shortcoming of the FBLM1 model by imposing the constraints on two different links. 


\section{Two-phase hybrid optimization approach: Path and motion generation syntheses}

The two-phase hybrid approach consists in synthesizing two separate linkages for path and motion generation of the jaw and then integrating both subordinate mechanisms into the final setup. To this end, two different methods will be used to synthesize each sub-mechanism: 1) The numerical optimization approach is used for the path generation and 2) the analytical synthesis approach is adopted for the motion generation. Indeed, the proposed design is a marriage of a four-bar linkage mechanism (FBLM-2) to generate the desirable trajectory of point $C$ and a three-rotational-joint (R) sub-mechanism called RRR-dyad to control the orientation of the jaw during its travel. The resulting mechanism would be a six-bar linkage mechanism called SBLM as illustrated in Fig. 5c.

\section{Path generation optimization of FBLM-2}

FBLM-2 is a four bar closed mechanism composed of the links $l_{1}$ through $l_{4}$ as shown in Fig. 5b. Four out of five constraints mentioned in the previous section are imposed to FBLM-2. In fact, constraint \#1 to constraint \#4 are all applied to FBLM-2, but constraint \#5 is relaxed to make the generated path closer to the target path for the point $C$. Except for constraints, the optimization procedure of FBLM-2 model remains quite similar to FBLM-1 with the same computational process, objective function and optimization algorithm.

\section{Analytical motion generation synthesis of RRR-dyad}

RRR-dyad is an open linkage consisting of the links $l_{5}$ and $l_{6}$ as shown in Fig. 5a. RRR-dyad is coupled up to the FBLM-2 via the joint located at $C$. This point represents the TMJ and $l_{6}$ constitutes the jaw. The RRR-dyad makes the jaw link $l_{6}$ pass through three specified positions of the point $C$ along the TMJ trajectory while controlling its orientation with the rotation angles $\alpha_{2}$ and $\alpha_{3}$ at the intermediate and final positions.

The analytical synthesis of RRR-dyad for three precision positions is based on the algebraic analysis of the vector loops formed by its links in the starting, intermediate and final positions. Therefore, three distinct positions of the point $C$ along the TMJ trajectory are chosen and the RRR-dyad model is schematically built at these positions as demonstrated in Fig. 5a.

The design variables including $l_{5}$ and $l_{6}$, their initial angular positions, $\theta_{\mathrm{O}, 0}$ and $\theta_{\mathrm{M}, 0}$, their intermediate and final rotations $\alpha_{2}, \alpha_{3}, \beta_{2}$ and $\beta_{3}$ with respect to their initial angular positions and the magnitude and orientation of the displacement vectors $p_{2}, p_{3}, \delta_{2}$ and $\delta_{3}$ to reach the intermediate and final positions can be identified in the model. Two vector loops are formed in Fig. 5a resulting in two vector equations as follow:

$$
\begin{aligned}
\overrightarrow{O M_{1}}+\overrightarrow{M_{1} C_{1}}+\overrightarrow{C_{1} C_{\frac{N}{2}}}+\overrightarrow{C_{\frac{N}{2}} M_{\frac{N}{2}}}+\overrightarrow{M_{\frac{N}{2}} O}=0 \\
\overrightarrow{O M_{1}}+\overrightarrow{M_{1} C_{1}}+\overrightarrow{C_{1} C_{N}}+\overrightarrow{C_{N} M_{N}}+\overrightarrow{M_{N} O}=0
\end{aligned}
$$

By decomposing the vector equations into two orthogonal scalar equations along $\vec{x}$ and $\vec{y}$, one can obtain:

$$
\left\{\begin{aligned}
l_{5} & \cos \theta_{\mathrm{O}}\left(\cos \beta_{2}-1\right)-l_{5} \sin \theta_{\mathrm{O}} \sin \beta_{2} \\
& \quad+l_{6} \cos \theta_{\mathrm{M}}\left(\cos \alpha_{2}-1\right)-l_{6} \sin \theta_{\mathrm{M}} \sin \alpha_{2}=p_{2} \cos \delta_{2} \\
& l_{5} \sin \theta_{\mathrm{O}}\left(\cos \beta_{2}-1\right)-l_{5} \cos \theta_{\mathrm{O}} \sin \beta_{2} \\
& +l_{6} \sin \theta_{\mathrm{M}}\left(\cos \alpha_{2}-1\right)-l_{6} \cos \theta_{\mathrm{M}} \sin \alpha_{2}=p_{2} \sin \delta_{2}
\end{aligned}\right.
$$

$$
\left\{\begin{array}{l}
l_{5} \cos \theta_{\mathrm{O}}\left(\cos \beta_{3}-1\right)-l_{5} \sin \theta_{\mathrm{O}} \sin \beta_{3} \\
\quad+l_{6} \cos \theta_{\mathrm{M}}\left(\cos \alpha_{3}-1\right)-l_{6} \sin \theta_{\mathrm{M}} \sin \alpha_{3}=p_{3} \cos \delta_{3} \\
l_{5} \sin \theta_{\mathrm{O}}\left(\cos \beta_{3}-1\right)-l_{5} \cos \theta_{\mathrm{O}} \sin \beta_{3} \\
\quad+l_{6} \sin \theta_{\mathrm{M}}\left(\cos \alpha_{3}-1\right)-l_{6} \cos \theta_{\mathrm{M}} \sin \alpha_{3}=p_{3} \sin \delta_{3}
\end{array}\right.
$$

The system of equations contains 12 unknowns for 4 equations. The 4 variables related to the point $C$ that is $p_{2}$, $\delta_{2}, p_{3}$ and $\delta_{3}$ are defined by the generated path obtained from the optimized FBLM-2 model. Moreover, according to the constraint \#5, the link $l_{6}$ should rotate $\Phi_{\text {hinge }}$ between the initial and final positions resulting in $\Phi_{\text {hinge }} / 2$ between the initial and the intermediate position $\frac{N}{2}$; so $\alpha_{2}$ and $\alpha_{3}$ are determined by

$$
\left\{\begin{array}{l}
\alpha_{2}=\Phi_{\text {hinge }} / 2=-16^{\circ} \\
\alpha_{3}=\Phi_{\text {hinge }}=-32^{\circ}
\end{array}\right.
$$

Furthermore, $\beta_{2}$ and $\beta_{3}$ are chosen arbitrarily. The system of Eqn. (14) and (15) can be presented in the matrix form by

$$
\left[\begin{array}{cccc}
A & -B & C & -D \\
F & -G & H & -K \\
B & A & D & C \\
G & F & K & H
\end{array}\right]\left\{\begin{array}{l}
l_{5 x} \\
l_{5 y} \\
l_{6 x} \\
l_{6 y}
\end{array}\right\}=\left\{\begin{array}{c}
E \\
L \\
M \\
N
\end{array}\right\}
$$

in which

$$
\begin{array}{lll}
l_{5 x}=l_{5} \cos \theta_{O} & A=\cos \beta_{2}-1 & G=\sin \beta_{3} \\
l_{5 y}=l_{5} \sin \theta_{O} & B=\sin \beta_{2} & H=\cos \alpha_{3}-1 \\
l_{6 x}=l_{6} \cos \theta_{M} & C=\cos \alpha_{2}-1 & K=\sin \alpha_{3} \\
l_{6 y}=l_{6} \sin \theta_{M} & D=\sin \alpha_{2} & L=p_{3} \cos \delta_{3} \\
& E=p_{2} \cos \delta_{2} & M=p_{2} \sin \delta_{2} \\
& F=\cos \beta_{3}-1 & N=p_{3} \sin \delta_{3}
\end{array}
$$




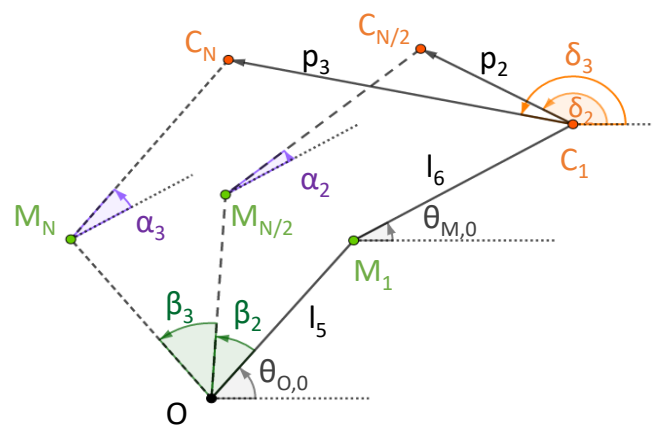

(a) RRR-dyad model

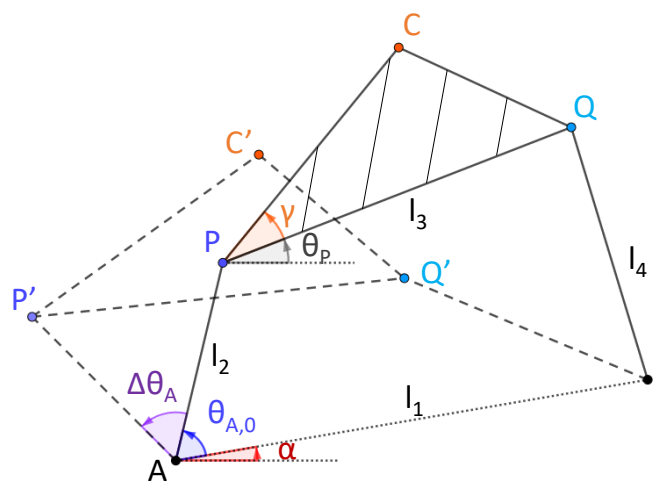

(b) FBLM-2 model

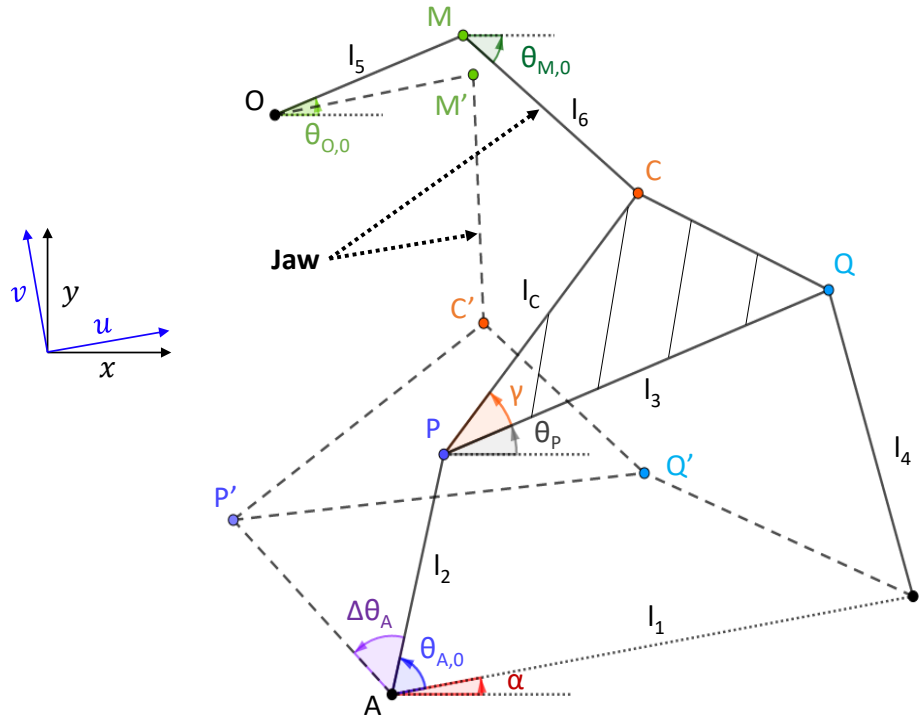

(c) SBLM model

Fig. 5: Schematic representation of (a) RRR-dyad model, (b) FBLM-2 model and (c) SBLM model obtained by attaching (a) and (b) from the point $C$. The initial positions are in solid line.

The solution of the matrix equation gives the link size variables $l_{5 x}, l_{5 y}, l_{6 x}$ and $l_{6 y}$. By knowing the position of the point $C$, one can find the position of the fixed pivot $O$ by:

$$
\left\{\begin{array}{l}
x_{O}=x_{C_{1}}-l_{6 x}-l_{5 x} \\
y_{O}=y_{C_{1}}-l_{6 y}-l_{5 y}
\end{array}\right.
$$

Finally, the analytically synthesized RRR-dyad submechanism can be integrated with the optimized FBLM-2 to form the six-bar mechanism SBLM capable of generating both the path and the motion of the jaw link during its travel from closed to open jaw position. The process of the two-phase hybrid approach is resumed in Fig. 6.

\section{Results}

Two-phase hybrid optimization approach is executed for ten different starting points in both configurations like the one-phase approach. The lowest obtained RMSE for the converged solution is calculated to be $\varepsilon=1.29 \%$ for the open configuration with the optimized set of design variables detailed in Table 2. Moreover, the first-order optimality value is equal to 0.015 which demonstrates a significant improvement over the results obtained for the classical one-phase optimization. In addition, the maximum constraint violation

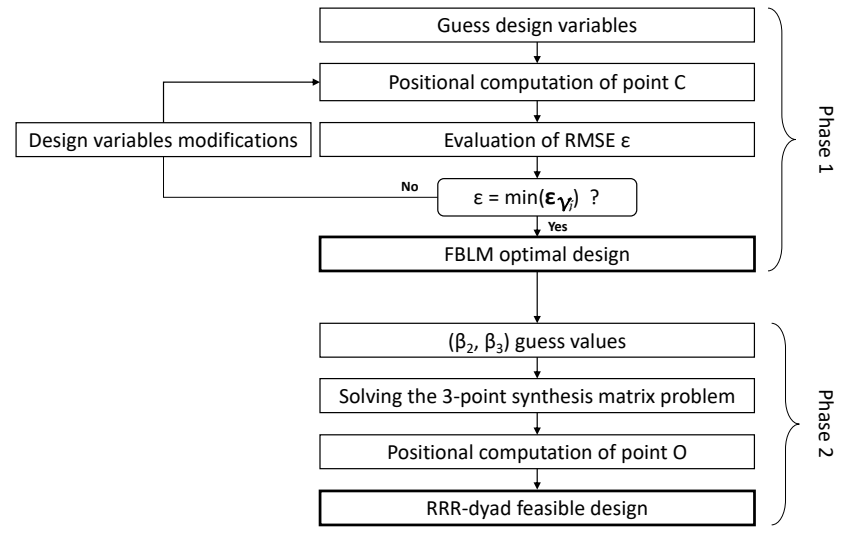

Fig. 6: Flowchart of the process for solving the two-phase hybrid optimization-analytical problem for FBLM-2 and RRR-dyad models. $\varepsilon_{V_{i}}$ : set of RMSE for the neighborhood of evaluation points at iteration ' $i$ '.

value is as low as $4.12 \times 10^{-5}$ which guarantees the satisfaction of all the imposed constraints.

The RRR-dyad analytical synthesis for the motion generation of the jaw link is highly dependent on the arbitrary values of $\beta_{2}$ and $\beta_{3}$. Therefore many couple values of $\left(\beta_{2}\right.$, $\beta_{3}$ ) are tested for the RRR-dyad and the obtained feasible 
Table 2: Optimized values of the design variables for the FBLM-2 model

\begin{tabular}{c|cc}
\hline$l_{1}$ & 137.72 & $\mathrm{~mm}$ \\
\hline$l_{2}$ & 24.84 & $\mathrm{~mm}$ \\
\hline$l_{3}$ & 26.94 & $\mathrm{~mm}$ \\
\hline$l_{4}$ & 137.68 & $\mathrm{~mm}$ \\
\hline$l_{C}$ & 34.47 & $\mathrm{~mm}$ \\
\hline$\alpha$ & $111.40^{\circ}$ & \\
\hline$\gamma$ & $14.04^{\circ}$ & \\
\hline$\theta_{A, O}$ & $185.16^{\circ}$ & \\
\hline$\Delta \theta_{A}$ & $108.42^{\circ}$ & \\
\hline$x_{A}$ & 17.53 & $\mathrm{~mm}$ \\
\hline$y_{A}$ & 3.62 & $\mathrm{~mm}$ \\
\hline & &
\end{tabular}

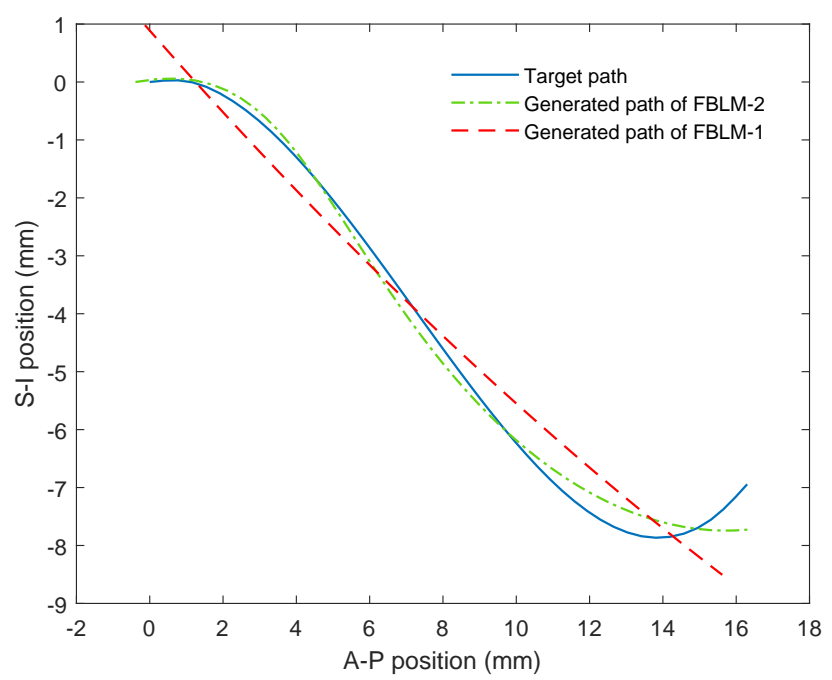

Fig. 7: Optimized generated path for the FBLM-2 model compared with the FBLM-1 and the target path. Closed jaw position corresponds to the origin of the coordinate system.

designs are marked. Among marked designs, several candidates are chosen based on the magnitude of the length and rotation of the links to minimize the work space of the mechanism. Finally, the best design is chosen by evaluating the key characteristic criterion of the jaw link rotation which is the smooth and uniform transition between the initial closed and the final open jaw positions. The values of the chosen angles are $\beta_{2}=-25^{\circ}$ and $\beta_{3}=-35^{\circ}$ and the associated values of design variables are listed in Table 3.

The optimized design of FBLM-2 and analytical synthesis of RRR-dyad are subsequently joined at the point $C$ to form the SBLM model. Finally, the digital mockup of
Table 3: Values of the design variables for the analytical solution of the RRR-dyad model

\begin{tabular}{c|cc}
\hline$l_{5}$ & 17.06 & $\mathrm{~mm}$ \\
\hline$l_{6}$ & 34.05 & $\mathrm{~mm}$ \\
\hline$\theta_{O, 0}$ & $6.64^{\circ}$ & \\
\hline$\theta_{M, 0}$ & $113.19^{\circ}$ & \\
\hline$x_{O}$ & -3.93 & $\mathrm{~mm}$ \\
\hline$y_{O}$ & -33.27 & $\mathrm{~mm}$ \\
\hline
\end{tabular}

the optimally synthesized six-bar linkage mechanism shown in Fig. 8 is provided using the Computer-Assisted Design (CAD) software CATIA V5 (Dassault Systèmes, VélizyVillacoublay, France). This virtual prototype enables verifying global kinematics of the mechanism, and validating the effectiveness of the proposed hybrid approach to replicate the motion of the jaw as shown in Fig. 9.

\section{Discussion}

According to Table 2, the length of all the links is less than $150 \mathrm{~mm}$ as required by the constraint \#1, the Grashof condition is satisfied as imposed by constraint \#2 and $l_{2}$ is the shortest link as forced by the constraint \#3. Therefore, the global size of the mechanism remains in an acceptable range. The optimized generated path of FBLM-2 (dash-dotted line) is illustrated in Fig. 7 and compared to the FBLM-1 (dashed line) and the target path (solid line).

According to Fig. 7, the global shape of the FBLM-2 curve is much closer to the target path than the FBLM-1. Particularly, an inflection point and S-shape form can be explicitly observed for the FBLM-2 curve in contrast to the FBLM-1. Furthermore, the path generated by FBLM-2 is more successful in pursuing the target path specifically at both extremities of the curves. Since the most of interactions between the earcanal and TMJ occurs at the end of the curve, the accuracy of the generated path at this location is very crucial. The overall improvement achieved by the proposed two-phase hybrid optimization over the classical single phase optimization can be quantified by comparing their associated errors which reveals two times better performance for the FBLM-2. The main reason is that the combined path and motion generation synthesis is overconstrained as for the FBLM-1 model, whereas the FBLM-2 model is properly constrained by assigning the motion generation constraint to the RRR-dyad submechanism. Therefore, the maximum constraint violation value is remarkably dropped and becomes almost zero for the path generation synthesis of FBLM-2.

The rotation of the jaw link $l_{6}$ in RRR-dyad is calculated while the TMJ is traveling along the trajectory curve and the results are demonstrated in Fig. 10. According to this figure, the angular variation of the jaw link is almost 

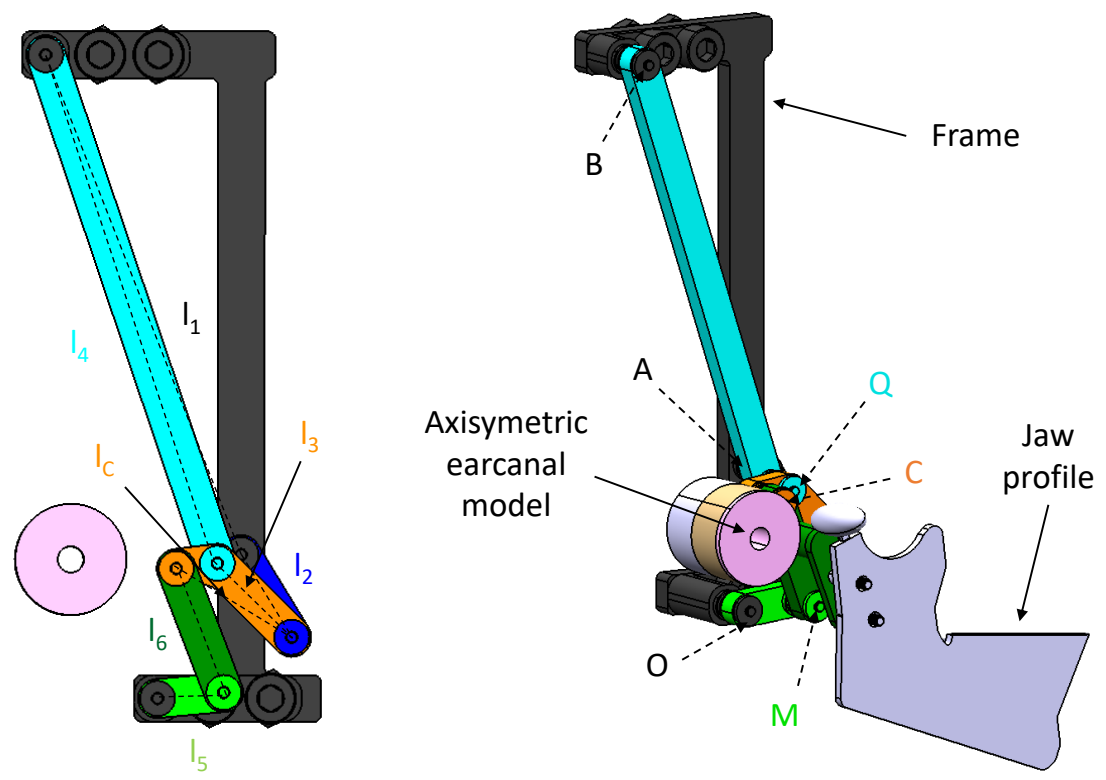

Fig. 8: Digital mockup of the optimized six-bar linkage mechanism and an axisymmetric earcanal model interacting with TMJ

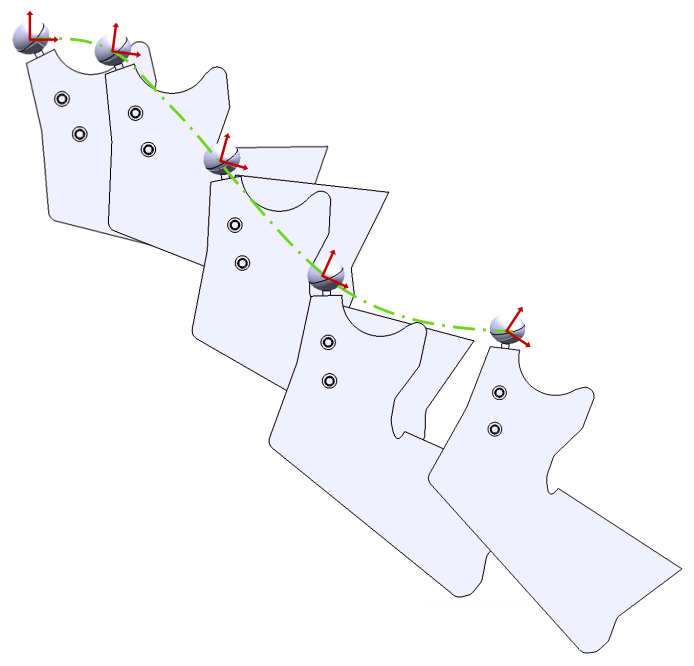

(a) Enlarged scale trajectory

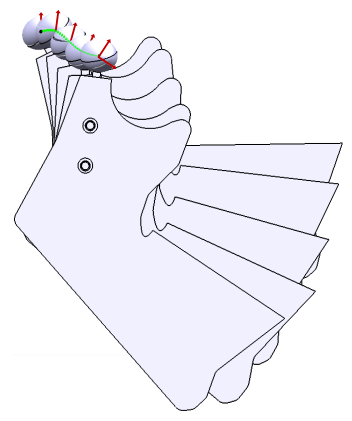

(b) Full scale trajectory

Fig. 9: Jaw motion generated by the SBLM model along the TMJ path (green line) linear with a constant rate of $2^{\circ}$ per millimeter of the TMJ displacement. From the kinematic point of view, it means a quasi-homogeneous movement for the mechanism. From the anatomic point of view, it means that the jaw rotation is quite uniform during the opening phase of the mouth. The translation-rotation correlation of the jaw has been already investigated by other researchers. For example, the contribution of the jaw rotation is estimated to be $2.1 \pm 0.2^{\circ}$ per millimeter of the TMJ translation [10] showing a difference of about $4.8 \%$ compared to what reported in this research. The difference can be explained by (1) the difference in measuring the horizontal translation of the TMJ $(15.0 \pm 1.6 \mathrm{~mm}$ versus $16 \mathrm{~mm}$ used in this work) and (2) the jaw opening rotation around the hinge axis $\left(31.9 \pm 4.2^{\circ}\right.$ versus $32^{\circ}$ in this work).

As both optimization approaches are dependent on the initial guess for the design variables, the validity of the optimized designs is limited to the vicinity of the initial guess set. To find the global optimal solutions, all the possibilities of initial guess need to be verified.

It should be noted that the human anatomy is not unique and hence, the kinematic parameters for describing the opening mouth movement may vary among individuals. Three well-individualised kinematic TMJ models correlated with temporal bone geometries have been reported in the literature and it is estimated that the inter-individual variation of the parameters is weak within each group [10]. All the results obtained in this study are closely dependent on the TMJ model described in section 2 .

However, the proposed synthesis approach could make it possible to customize the SBLM generated path to model more than one TMJ anatomy. The solving process detailed in Fig. 6 would remain the same, and only the biomechanical constraints values would be adjusted according to the 


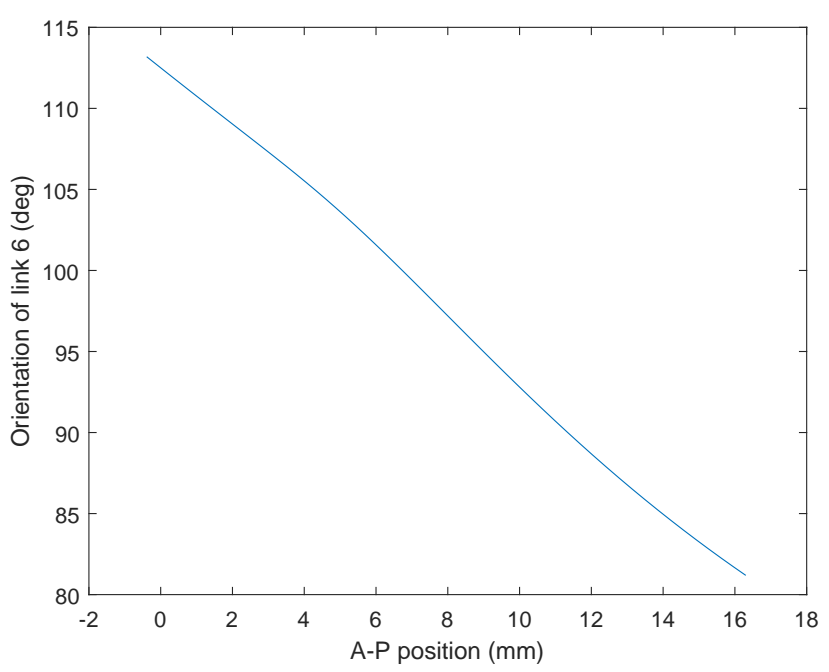

Fig. 10: Angular orientation of the jaw link $l_{6}$ according to the A-P position

TMJ anatomical model. In add, the mockup design could integrate threaded rods, adjustment spacers and a compasslike coupler. It could make it possible to adjust all the link lengths and the coupler angle in order to take into account the variablity of the TMJ anatomy.

\section{Conclusions}

A six-bar kinematic simulator consisting of a four-bar mechanism and a RRR-dyad linkage has been presented in this paper to generate the path of the TMJ and the motion of the jaw for a typical jaw opening movement.

The complexity of the objective function to minimize the error between the anatomical target path and the mechanism generated curve as well as the strong nonlinear constraints imposed by the anatomy of the jaw movements makes the solution consisting of a simple four-bar linkage mechanism (FBLM-1) insufficient. Correspondingly, the associated design problem became overconstraint and the classical single phase optimization approach could not return an acceptable converged solution. Therefore, the two-phase optimization approach was proposed for splitting the path and motion generation of the jaw movement between two different submechanisms. Subsequently, the solution of nonlinear optimization for a properly constrained four-bar linkage mechanism (FBLM-2) was integrated into an analytical solution for three-position synthesis of a two-link dyad (RRRdyad) to successfully provide a six-bar linkage mechanism capable of reproducing both the TMJ path and the jaw motion while respecting all the imposed constraints.

The proposed two-phase optimization approach can be further used to design linkage mechanisms in general and human prosthesis in particular where complicated objective functions and highly nonlinear constraints impair using the classical optimization approach. Moreover, the developed kinematic simulator would facilitate the morphological study of TMJ and the deformation analysis of earcanal while providing a platform to validate the kinematic mechanisms, biomechanical computer simulations or finite element models of the TMJ-earcanal interactions. Additionally, the proposed simulator can give new insights into the complex motion of the TMJ, improve the mandibular implant design and eventually lead to understand and treat the temporomandibular joint (TMJ) diseases. Furthermore, it can enable to investigate the discomfort issues related to hearing prostheses and simplify the evaluation of the kinetic energy associated with earcanal dynamic movements for energy harvesting.

\section{Acknowledgements}

The authors would like to acknowledge the support of the Natural Sciences and Engineering Council of Canada (NSERC), through last author's Discovery grant (RGPIN2017-06192). The technical support received from the NSERC-EERS Industrial Research Chair in In-Ear Technologies (CRITIAS) was also greatly appreciated.

\section{References}

[1] Delnavaz, A., and Voix, J., 2013. "Ear canal dynamic motion as a source of power for in-ear devices". Journal of Applied Physics, 113(6), Feb., p. 9 p. 00003.

[2] Bouchard-Roy, J., Delnavaz, A., and Voix, J., 2020. "In-Ear Energy Harvesting: Evaluation of the Power Capability of the Temporomandibular Joint". IEEE Sensors Journal, 20(12), June, pp. 6338-6345. Conference Name: IEEE Sensors Journal.

[3] Carioli, J., Delnavaz, A., Zednik, R. J., and Voix, J., 2016. "Power capacity from earcanal dynamic motion". AIP Advances, 6(12), Dec., p. 125203. 00000.

[4] Travers, K. H., Buschang, P. H., Hayasaki, H., and Throckmorton, G. S., 2000. "Associations between incisor and mandibular condylar movements during maximum mouth opening in humans". Archives of Oral Biology, 45(4), Apr., pp. 267-275.

[5] Koolstra, J., Naeije, M., and Van Eijden, T., 2001. "The Three-dimensional Active Envelope of Jaw Border Movement and its Determinants". Journal of Dental Research, 80(10), Oct., pp. 1908-1912.

[6] Azuma, T., Ito, J., Kutsuki, M., Nakai, R., Fujita, S., and Tsutsumi, S., 2009. "Analysis of the mandibular movement by simultaneous multisection continuous ultrafast MRI". Magnetic Resonance Imaging, 27(3), Apr., pp. 423-433.

[7] Baeyens, J.-P., Gilomen, H., Erdmann, B., Clijsen, R., Cabri, J., and Vissers, D., 2013. "In vivo measurement of the 3D kinematics of the temporomandibular joint using miniaturized electromagnetic trackers: technical report". Medical \& Biological Engineering \& Computing, 51(4), Apr., pp. 479-484.

[8] Yatabe, M., Zwijnenburg, A., Megens, C., and Naeije, M., 1997. "Movements of the Mandibular Condyle Kinematic Center during Jaw Opening and Closing”. Journal of Dental Research, 76(2), Feb., pp. 714-719. 
[9] Ahn, S.-J., Tsou, L., Antonio Sánchez, C., Fels, S., and Kwon, H.-B., 2015. "Analyzing center of rotation during opening and closing movements of the mandible using computer simulations". Journal of Biomechanics, 48(4), Feb., pp. 666-671.

[10] Mesnard, M., Coutant, J. C., Aoun, M., Morlier, J., Cid, M., and Caix, P., 2012. "Relationships between geometry and kinematic characteristics in the temporomandibular joint". Computer Methods in Biomechanics and Biomedical Engineering, 15(4), Apr., pp. 393-400.

[11] Chen, C.-C., Lin, C.-C., Lu, T.-W., Chiang, H., and Chen, Y.-J., 2013. "Feasibility of differential quantification of 3D temporomandibular kinematics during various oral activities using a cone-beam computed tomography-based 3D fluoroscopic method". Journal of Dental Sciences, 8(2), June, pp. 151-159.

[12] Gallo, L., Airoldi, G., Airoldi, R., and Palla, S., 1997. "Description of Mandibular Finite Helical Axis Pathways in Asymptomatic Subjects". Journal of Dental Research, 76(2), Feb., pp. 704-713.

[13] Hayashi, K., Hayashi, M., Reich, B., Lee, S.-P., Sachdeva, A. U. C., and Mizoguchi, I., 2012. "Functional data analysis of mandibular movement using third-degree b-spline basis functions and self-modeling regression". Orthodontic Waves, 71(1), Mar., pp. 1725.

[14] Takanobu, H., Takanishi, A., and Kato, I., 1994. "Control of a mastication robot for reduction of jaw joint force focusing on musculus temporalis". In Proceedings of IEEE/RSJ International Conference on Intelligent Robots and Systems (IROS'94), Vol. 3, IEEE, pp. 1824-1831.

[15] Takanishi, A., Tanase, T., Kumei, M., and Kato, I., 1991. "Development of 3 DOF jaw robot WJ-2 as a human's mastication simulator". IEEE Xplore.

[16] Takanobu, H., Yajima, T., Nakazawa, M., Takanishi, A., Ohtsuki, K., and Ohnishi, M., 1998. "Quantification of masticatory efficiency with a mastication robot". In Proceedings. 1998 IEEE International Conference on Robotics and Automation (Cat. No.98CH36146), Vol. 2, IEEE, pp. 1635-1640.

[17] Galer, B., Hockenberry, N., Maloof, J., and Montelowry, M., 2007. Human Jaw Motion Simulator. Apr.

[18] Tahir, A. M., Jilich, M., Trinh, D. C., Cannata, G., Barberis, F., and Zoppi, M., 2019. "Architecture and design of a robotic mastication simulator for interactive load testing of dental implants and the mandible". The Journal of Prosthetic Dentistry, 122(4), Oct., pp. 389.e1389.e8.

[19] Mostashiri, N., Dhupia, J., Verl, A., Bronlund, J., and $\mathrm{Xu}, \mathrm{W} ., 2020$. "Optimizing the Torque Distribution of a Redundantly Actuated Parallel Robot to Study the Temporomandibular Reaction Forces During Food Chewing”. Journal of Mechanisms and Robotics, 12(5), Oct. Publisher: American Society of Mechanical Engineers Digital Collection.

[20] Pirzanski, C., and Berge, B., 2005. "Ear canal dynamics: Facts versus perception". The Hearing Journal,
58(10), Oct., p. 50.

[21] Pirzanski, C., 2010. "Ear mold retention issues: Why do these ear molds keep falling out". Hearing Review, 17(5), pp. 26-34.

[22] Nielsen, C., and Darkner, S., 2011. "The cartilage bone junction and its implication for deep canal hearing instrument fittings". The Hearing Journal, 64(3), pp. 3536.

[23] Haulin, E. N., Lakis, A. A., and Vinet, R., 2001. "Optimal synthesis of a planar four-link mechanism used in a hand prosthesis". Mechanism and Machine Theory, 36(11), Nov., pp. 1203-1214.

[24] Tsuge, B. Y., Plecnik, M. M., and Michael McCarthy, J., 2016. "Homotopy Directed Optimization to Design a Six-Bar Linkage for a Lower Limb With a Natural Ankle Trajectory". Journal of Mechanisms and Robotics, 8(6), Dec. Publisher: American Society of Mechanical Engineers Digital Collection.

[25] Guo, G., Zhang, J., and Gruver, W. A., 1993. “Optimal Design of a Six-Bar Linkage with One Degree of Freedom for an Anthropomorphic Three-Jointed Finger Mechanism". Proceedings of the Institution of Mechanical Engineers, Part H: Journal of Engineering in Medicine, 207(3), Sept., pp. 185-190.

[26] Doutres, O., Sgard, F., Terroir, J., Perrin, N., Jolly, C., Gauvin, C., and Negrini, A., 2019. "A critical review of the literature on comfort of hearing protection devices: definition of comfort and identification of its main attributes for earplug types". International Journal of Audiology, 58(12), Dec., pp. 824-833.

[27] Kochkin, S., 2000. "MarkeTrak V: "Why my hearing aids are in the drawer": The consumers' perspective". The Hearing Journal, 53(2), p. 5.

[28] Elliott H. Berger, and Jérémie Voix, 2018. "Hearing Protection Devices". In The Noise Manual, Meinke, DK, Berger, EH, Neitzel, R, Driscoll, DP, and Hager, LD, eds., 6th edition ed. American Industrial Hygiene Association. 00177.

[29] Delnavaz, A., and Voix, J., 2014. "Energy harvesting for in-ear devices using ear canal dynamic motion". 00014.

[30] Delnavaz, and Voix, 2014. "Flexible piezoelectric energy harvesting from jaw movements". Smart Materials and Structures - IOP Publishing Ltd, Vol. 23(Num. 10), Oct., p. 8 pp. 00015.

[31] Carioli, J., Delnavaz, A., Zednik, R. J., and Voix, J., 2018. "Piezoelectric Earcanal Bending Sensor". IEEE Sensors Journal, 18(5), Mar., pp. 2060-2067.

[32] Peck, C. C., Langenbach, G. E. J., and Hannam, A. G., 2000. "Dynamic simulation of muscle and articular properties during human wide jaw opening". Archives of Oral Biology, 45(11), Nov., pp. 963-982.

[33] Simón Mata, A., Bataller Torras, A., Cabrera Carrillo, J. A., Ezquerro Juanco, F., Guerra Fernández, A. J., Nadal Martínez, F., and Ortiz Fernández, A., 2016. Fundamentals of Machine Theory and Mechanisms, Vol. 40 of Mechanisms and Machine Science. Springer International Publishing, Cham. 